\title{
Correction to: Biodiversity and Health in the Urban Environment
}

\author{
Melissa R. Marselle ${ }^{1}$ - Sarah J. Lindley ${ }^{2}$ - Penny A. Cook ${ }^{3}$ - Aletta Bonn ${ }^{4,5,6}$
}

Published online: 1 July 2021

(C) Springer Nature Switzerland AG 2021

\section{Correction to: Current Environmental Health Reports (2021)} https://doi.org/10.1007/s40572-021-00313-9

Following the publication of the original article, the publisher regrets a mistake in one of the author's affiliations. The affiliations for co-author Aletta Bonn should be:

* Department of Ecosystem Services, Helmholtz Centre for Environmental Research - UFZ, Permoserstr. 15, 04318 Leipzig, Germany

* Institute of Biodiversity, Friedrich Schiller University Jena, Dornburger Straße 159, 07743 Jena, Germany

* Department of Ecosystem Services, German Centre for Integrative Biodiversity Research (iDiv), Halle-Jena-Leipzig, Puschstr. 4, 04103 Leipzig, Germany

The original article has been corrected.

Publisher's Note Springer Nature remains neutral with regard to jurisdictional claims in published maps and institutional affiliations.

The online version of the original article can be found at https://doi.org/ 10.1007/s40572-021-00313-9

Melissa R. Marselle

m.marselle@surrey.ac.uk

Sarah J. Lindley

sarah.lindley@manchester.ac.uk

Penny A. Cook

P.A.Cook@salford.ac.uk

Aletta Bonn

aletta.bonn@idiv.de

1 School of Psychology, University of Surrey, Guildford GU2 7XH, UK
2 Department of Geography, School of Environment, Education and Development, University of Manchester, Oxford Road, Manchester M13 9PL, UK

3 School of Health and Society, University of Salford, M6 6PU, Salford, UK

4 Department of Ecosystem Services, Helmholtz Centre for Environmental Research - UFZ, Permoserstr. 15, 04318 Leipzig, Germany

5 Institute of Biodiversity, Friedrich Schiller University Jena, Dornburger Straße 159, 07743 Jena, Germany

6 Department of Ecosystem Services, German Centre for Integrative Biodiversity Research (iDiv) Halle-Jena-Leipzig, Puschstr. 4, 04103 Leipzig, Germany 